\title{
Applications of Fixed Point Theorems to Generalized Saddle Points of Bifunctions on Chain-Complete Posets
}

\author{
Jinlu Li, ${ }^{1}$ Ying Liu, ${ }^{2}$ and Hongya Gao ${ }^{2}$ \\ ${ }^{1}$ Department of Mathematics, Shawnee State University, Portsmouth, OH 45662, USA \\ ${ }^{2}$ College of Mathematics and Computer, Hebei University, Baoding, Hebei 071002, China \\ Correspondence should be addressed to Ying Liu; ly_cyh2013@163.com
}

Received 15 January 2014; Accepted 14 March 2014; Published 9 April 2014

Academic Editor: Chong Li

Copyright (c) 2014 Jinlu Li et al. This is an open access article distributed under the Creative Commons Attribution License, which permits unrestricted use, distribution, and reproduction in any medium, provided the original work is properly cited.

We apply the extensions of the Abian-Brown fixed point theorem for set-valued mappings on chain-complete posets to examine the existence of generalized and extended saddle points of bifunctions defined on posets. We also study the generalized and extended equilibrium problems and the solvability of ordered variational inequalities on posets, which are equipped with a partial order relation and have neither an algebraic structure nor a topological structure.

\section{Introduction}

Let $X$ be a topological vector space and let $C$ be a subset of $X$. Let $F$ be a real valued function defined on $C \times C$. A point $\left(x^{*}, y^{*}\right) \in C \times C$ is called a saddle point of the function $F$ if it satisfies

$$
F\left(x, y^{*}\right) \leq F\left(x^{*}, y^{*}\right) \leq F\left(x^{*}, y\right), \quad \forall x \in y \in C .
$$

A point $\left(x^{\prime}, y^{\prime}\right) \in C \times C$ is called an equilibrium of the function $F$ if it satisfies

$$
F\left(x, y^{\prime}\right) \geq F\left(x^{\prime}, y^{\prime}\right), \quad \forall x \in C .
$$

In optimization theory, the standard tools for dealing with the existence of saddle points and equilibria of a bifunction $F$ are fixed point theorems with respect to set-valued mappings, Fan-KKM theorem, variational inequalities, or others, where the considered bifunction $F$ must hold a certain type of continuity properties and the underlying subset $C$ must satisfy some geometrical conditions, such as convexity. The problems (SP) and (EP) have been extensively studied by many authors in many fields, such as variational inequality theory, complementarity problem theory, fixed point theory, convex analysis with applications to economic theory, and game theory (see [1-6]).

The concepts defined in (SP) and (EP) have been generalized by Giannessi [7], in 1980, to the cases that the income spaces of mappings are finite-dimensional vector spaces and the outcome spaces are ordered vector spaces that are posets equipped with both topological structure and algebraic structure. The extended problems are called vector optimization problems and vector variational inequalities. Since the underlying spaces in these problems are still equipped with both topological structure and algebraic structure, then they may also be solved by the standard techniques as in solving problems (SP) and (EP).

In economic theory and social sciences, there are some examples that both of the income and outcome spaces of mappings are posets, which are equipped with neither topological structure nor algebraic structure. Then, in these circumstances, the optimization problems will be orderoptimization problems, which are not normal optimization problems (with respect to real valued functions) and they cannot be solved by using the standard methods. The saddle points and equilibria of bifunction must be generalized from real valued functions to functions with values in ordered sets. In this case, more fixed point theorems on ordered sets must be acquired and some new analysis techniques dealing with mappings on ordered sets must be developed.

In [8], several extensions of the Abian-Brown fixed point theorem provided in [9] on posets are obtained, which are extensions from single valued mappings to set-valued mappings. Moreover, the author examined some nonmonetized 
noncooperative games where both the collections of the strategies and the ranges of the utilities for the players are posets and proved some existence theorems of extended and generalized Nash equilibria for nonmonetized, noncooperative games on chain-complete posets by applying the extensions.

On the other hand, in [10], Xie et al. generalized the extensions of the Abian-Brown fixed point theorem provided in [8] from chain-complete posets to chain-complete preordered sets for set-valued mappings. By using these generalizations and by applying the order-increasing upward property of set-valued mappings, they also prove several existence theorems of the extended and generalized Nash equilibria of nonmonetized noncooperative games on chaincomplete preordered sets.

References $[8,10]$ mainly considered the existence of the fixed point of set-valued mappings in chain-complete posets and chain-complete preordered sets, respectively. The applications of these fixed point theorems were considered less, although $[8,10]$ gave some examples of nonmonetized noncooperative games on posets and preordered sets. To remedy the defect, we apply the extensions of the AbianBrown fixed point theorem provided in [9] on posets to examine the existence of generalized and extended saddle points of bifunctions on posets. We also study the solvability of generalized and extended equilibrium problems of bifunctions and ordered variational inequalities on posets, which have neither an algebraic structure nor a topological structure.

\section{Several Extensions of the Abian-Brown Fixed Point Theorem on Posets}

The order-increasing property of mappings is important for the considered mappings to have a fixed point. In this section, we recall the notations of the order-increasing property of mappings, which are used in $[2,9,11,12]$.

Let $\left(X, \geqslant^{X}\right),\left(U, \succcurlyeq^{U}\right)$ be posets and let $F: X \rightarrow 2^{U} \backslash\{\varnothing\}$ be a set-valued mapping. $F$ is said to be isotone or to be orderincreasing upward, if $x \leqslant^{X} y$ in $X$; then, for any $z \in F(x)$, there is a $w \in F(y)$ such that $z \preccurlyeq^{U} w . F$ is said to be order-increasing downward, if $x \preccurlyeq^{X} y$ in $X$; then, for any $w \in F(y)$, there is a $z \in F(x)$ such that $z \preccurlyeq^{U} w$. If $F$ is both order-increasing upward and order-increasing downward, then $F$ is said to be order-increasing. As a special case, a single valued mapping $F$ from a poset $\left(X, \geqslant^{X}\right)$ to a poset $\left(U, \geqslant^{U}\right)$ is said to be orderincreasing whenever $x \leqslant^{X} y$ implies $F(x) \preccurlyeq^{U} F(y)$.

2.1. The Abian-Brown Fixed Point Theorem. Let $(P, \geqslant)$ be a chain-complete poset and let $F: P \rightarrow P$ be an orderincreasing single valued mapping. If there is an $x^{*}$ in $P$ with $F\left(x^{*}\right) \geqslant x^{*}$, then $F$ has a fixed point.

Similar to the well-known Kakutani contribution that extended the Brouwer fixed point theorem from single valued mappings to set-valued mappings in topological spaces, the main results of [8] extended the Abian-Brown fixed point theorem from single valued mappings to set-valued mappings in ordered sets, which are also the generalization of the Fujimoto-Tarski fixed point theorem from complete lattices to chain-complete posets. We recall the extensions obtained in [8] below.

Theorem 1. Let $(P, \geqslant)$ be a chain-complete poset and let $F: P \rightarrow 2^{P} \backslash\{\varnothing\}$ be a set-valued mapping. If $F$ satisfies the following three conditions,

(A1) $F$ is order-increasing upward;

(A2) the set $\{z \in P: z \leqslant u$, for some $u \in F(x)\}$ is an inductively ordered set, for each $x \in P$;

(A3) there is a $y$ in $P$ with $y \leqslant u$, for some $u \in F(y)$,

then $F$ has a fixed point; that is, there exists $x^{*} \in P$ such that $x^{*} \in F\left(x^{*}\right)$.

Theorem 2. Let $(P, \geqslant)$ be a chain-complete poset and let $F: P \rightarrow 2^{P} \backslash\{\varnothing\}$ be a set-valued mapping, which satisfies conditions (A1) and (A3) given in Theorem 1. If any one of the following properties holds,

$\left(\mathrm{A} 2^{\prime}\right)(F(x), \geqslant)$ is inductive with finite number of maximal elements, for every $x \in P$;

$\left(\mathrm{A} 2^{\prime \prime}\right)(F(x), \geqslant)$ has a maximum element, for every $x \in P$;

$\left(\mathrm{A} 2^{\prime \prime \prime}\right)(F(x), \geqslant)$ is a chain-complete lattice, for each $x \in P$,

then $F$ has a fixed point.

\section{Generalized and Extended Saddle Points of Bifunctions on Posets}

The generalized saddle points of bifunctions on Banach lattices were studied in [13]. In this section, we extend this concept to posets.

Definition 3. Let $\left(X, \succcurlyeq^{X}\right)$ and $\left(Y, \succcurlyeq^{Y}\right)$ be posets. Let $\succcurlyeq^{X \times Y}$ be the coordinate ordering relation on the Cartesian product $X \times Y$ induced by the partial orders $\geqslant^{X}$ and $\geqslant^{Y}$. That is, for any $\left(x_{1}, y_{1}\right)$ and $\left(x_{2}, y_{2}\right) \in X \times Y$,

$$
\left(x_{1}, y_{1}\right) \geqslant^{X \times Y}\left(x_{2}, y_{2}\right) \quad \text { iff } x_{1} \geqslant^{X} x_{2}, y_{1} \geqslant^{Y} y_{2} .
$$

The proof of the following lemma is straight forward and is omitted.

Lemma 4. Let $\left(X, \geqslant^{X}\right)$ and $\left(Y, \succcurlyeq^{Y}\right)$ be posets. The coordinate ordering $\geqslant^{X \times Y}$ on $X \times Y$ induced by the partial orders $\geqslant^{X}$ and $\geqslant^{Y}$ defines a partial ordering relation on $X \times Y$; and hence $(X \times$ $\left.Y, \geqslant^{X \times Y}\right)$ is a poset. Furthermore, if $\left(X, \geqslant^{X}\right)$ and $\left(Y, \geqslant^{Y}\right)$ are both (conditionally) chain-complete (inductive, Dedekind complete) posets, then $\left(X \times Y, \geqslant^{X \times Y}\right)$ is also a (conditionally) chaincomplete (inductive, Dedekind complete) poset.

Definition 5. Let $\left(X, \geqslant^{X}\right),\left(Y, \geqslant^{Y}\right)$, and $\left(U ; \geqslant^{U}\right)$ be posets. Let $C$ and $D$ be nonempty subsets of $X$ and $Y$, respectively. Let 
$F: C \times D \rightarrow U$ be a mapping. A point $\left(x^{*}, y^{*}\right) \in C \times D$ is called a generalized saddle point of the mapping $F$ if it satisfies

$$
F\left(x, y^{*}\right) \preccurlyeq^{U} F\left(x^{*}, y^{*}\right) \preccurlyeq^{U} F\left(x^{*}, y\right), \quad \forall x \in C, y \in D .
$$

Definition 6. Let $\left(X, \succcurlyeq^{X}\right),\left(Y, \succcurlyeq^{Y}\right)$, and $\left(U ; \succcurlyeq^{U}\right)$ be posets. Let $C$ and $D$ be nonempty subsets of $X$ and $Y$, respectively. Let $F: C \times D \rightarrow U$ be a mapping. $F(x, y)$ is said to be

(1) order-negative with respect to $x$, whenever for any $x_{2} \succcurlyeq^{X} x_{1}$ in $C, F\left(x_{1}, y\right) \preccurlyeq^{U} F\left(x_{1}, t\right)$ implies $F\left(x_{2}, y\right)$ $\preccurlyeq^{U} F\left(x_{2}, t\right)$, for any $y, t \in D$;

(2) order-positive with respect to $y$, whenever for any $y_{2} \geqslant^{Y} y_{1}$ in $C, F\left(x, y_{1}\right) \geqslant^{U} F\left(s, y_{1}\right)$ implies $F\left(x, y_{2}\right) \geqslant{ }^{U} F\left(s, y_{2}\right)$, for any $x, s \in C$.

For a given mapping $F: C \times D \rightarrow U$, one defines mappings $\Upsilon: C \rightarrow 2^{D}$ and $\Psi: D \rightarrow 2^{C}$ by

$$
\begin{aligned}
& \Upsilon(x)=\left\{t \in D: F(x, t)=\bigwedge_{y \in D} F(x, y)\right\}, \quad \text { for any } x \in C, \\
& \Psi(y)=\{s \in C: F(s, y)=\underset{x \in C}{\vee} F(x, y)\}, \quad \text { for any } y \in D .
\end{aligned}
$$

We prove the following theorem for the existence of generalized saddle point.

Theorem 7. Let $\left(X, \geqslant^{X}\right),\left(Y, \geqslant^{Y}\right)$, and $\left(U ; \succcurlyeq^{U}\right)$ be posets. Let $C$ and $D$ be nonempty chain-complete subsets of $X$ and $Y$, respectively. Let $F: C \times D \rightarrow U$ be a mapping. If $F$ satisfies the following conditions,

(1) $F(x, y)$ is order-negative with respect to $x \in C$;

(2) $F(x, y)$ is order-positive with respect to $y \in D$;

(3) for every fixed $x \in C,\left(\Upsilon(x), \geqslant^{Y}\right)$ is a nonempty inductive subset of $D$ with finite number of maximal elements;

(4) For every fixed $y \in D,\left(\Psi(y), \geqslant^{X}\right)$ is a nonempty inductive subset of $C$ with finite number of maximal elements;

(5) There is an element $\left(x^{\prime}, y^{\prime}\right) \in C \times D$ with $x^{\prime} \leqslant{ }^{X} s$, for some $s \in \Psi\left(y^{\prime}\right)$, and $y^{\prime} \leqslant^{Y} t$, for some $t \in \Upsilon\left(x^{\prime}\right)$,

then $F$ has a generalized saddle point.

Proof. From Lemma $4,\left(C \times D, \geqslant^{X \times Y}\right)$ is a chain-complete poset. Define $T: C \times D \rightarrow 2^{C \times D} \backslash\{\varnothing\}$ by

$$
T(x, y)=\Psi(y) \times \Upsilon(x), \quad \forall(x, y) \in C \times D .
$$

From conditions (3) and (4), $T$ is well defined.

Next we show that $T$ is order-increasing upward. To this end, we show that, for any $\left(x_{1}, y_{1}\right)$ and $\left(x_{2}, y_{2}\right) \in C \times D$, $\left(x_{2}, y_{2}\right) \geqslant^{X \times Y}\left(x_{1}, y_{1}\right)$ implies $T\left(x_{1}, y_{1}\right) \subseteq T\left(x_{2}, y_{2}\right)$. For any given $\left(s_{1}, t_{1}\right) \in T\left(x_{1}, y_{1}\right)$, we have $s_{1} \in \Psi\left(y_{1}\right)$ and $t_{1} \epsilon$ $\Upsilon\left(x_{1}\right)$; that is,

$$
\begin{aligned}
& F\left(s_{1}, y_{1}\right)=\bigvee_{x \in C} F\left(x, y_{1}\right), \\
& F\left(x_{1}, t_{1}\right)=\wedge_{y \in D} F\left(x_{1}, y\right) .
\end{aligned}
$$

From (4), we have $F\left(s_{1}, y_{1}\right) \geqslant{ }^{U} F\left(x, y_{1}\right)$, for all $x \in C$. Since $y_{2} \geqslant^{Y} y_{1}$, then, from condition (2) in this theorem that $F(x, y)$ is order-positive with respect to $y \in D$, it implies $F\left(s_{1}, y_{2}\right) \geqslant{ }^{U} F\left(x, y_{2}\right)$, for all $x \in C$. So

That is, $F\left(s_{1}, y_{2}\right)=\vee_{x \in C} F\left(x, y_{2}\right)$. We obtain $s_{1} \in \Psi\left(y_{2}\right)$.

$$
\Psi\left(y_{1}\right) \subseteq \Psi\left(y_{2}\right) .
$$

From (5), similar to the proof of (6), for $x_{2} \geqslant{ }^{X} x_{1}$, we can show that

$$
\Upsilon\left(x_{1}\right) \subseteq \Upsilon\left(x_{2}\right) .
$$

Combining (6) and (7), we get

$$
\Psi\left(y_{1}\right) \times \Upsilon\left(x_{1}\right) \subseteq \Psi\left(y_{2}\right) \times \Upsilon\left(x_{2}\right) .
$$

That is, $T\left(x_{1}, y_{1}\right) \subseteq T\left(x_{2}, y_{2}\right)$. It implies that $T$ is orderincreasing upward.

We claim that an element $(p, q)$ is a maximal element of $\Psi(y) \times \Upsilon(x)$, if and only if $p$ is a maximal element of $\Psi(y)$ and $q$ is a maximal element of $\Upsilon(x)$. In fact, if $p$ is a maximal element of $\Psi(y)$ and $q$ is a maximal element of $Y(x)$, then for any $(s, t) \in \Psi(y) \times \Upsilon(x)$, we have $p \geqslant{ }^{X} s$ or $p \bowtie^{X} s$ and $q \geqslant Y$ t or $q \bowtie^{X} t$, which imply $(p, q) \geqslant^{X \times Y}(s, t)$ or $(p, q) \bowtie^{X \times Y}(s, t)$. So $(p, q)$ is a maximal element of $\Psi(y) \times \Upsilon(x)$. On the other hand, if $p$ is not a maximal element of $\Psi(y)$ or $q$ is not a maximal element of $\Upsilon(x)$, say, $p$ is not a maximal element of $\Psi(y)$, then there is $s \in \Psi(y)$ with $s \succ^{X} p$; that is, $(s, q) \succ^{X \times Y}(p, q)$. It implies that $(p, q)$ cannot be a maximal element of $\Psi(y) \times \Upsilon(x)$. Hence, from Lemma 4 and conditions (3) and (4) in this theorem, $\left(T(x, y), \geqslant^{X \times Y}\right)$ is an inductive poset with finite number of maximal elements.

From condition (5) of this theorem, it is clearly seen that the element $\left(x^{\prime}, y^{\prime}\right) \in C \times D$ with $x^{\prime} \preccurlyeq^{X}$, for some $s \in \Psi\left(y^{\prime}\right)$ and $y^{\prime} \preccurlyeq^{Y} t$ and for some $t \in \Upsilon\left(x^{\prime}\right)$, satisfies

$$
(s, t) \in \Psi\left(y^{\prime}\right) \times \Upsilon\left(x^{\prime}\right)=T\left(x^{\prime}, y^{\prime}\right), \quad\left(x^{\prime}, y^{\prime}\right) \preccurlyeq^{X \times Y}(s, t) .
$$

Hence the mapping $T$ from $C \times D$ to $2^{C \times D} \backslash\{\varnothing\}$ satisfies all conditions in Theorem 2 with respect to condition $\left(\mathrm{A} 2^{\prime}\right)$.So $T$ has a fixed point; say $\left(x^{*}, y^{*}\right) \in C \times D$; that is, $\left(x^{*}, y^{*}\right) \in$ $T\left(x^{*}, y^{*}\right)$. Then we have $x^{*} \in \Psi\left(y^{*}\right)$ and $y^{*} \in \Upsilon\left(x^{*}\right)$. So

$$
F\left(x^{*}, y^{*}\right)=\underset{x \in C}{\vee} F\left(x, y^{*}\right), \quad F\left(x^{*}, y^{*}\right)=\underset{y \in D}{\wedge} F\left(x^{*}, y\right) .
$$

It is equivalent to

$$
F\left(x, y^{*}\right) \preccurlyeq^{U} F\left(x^{*}, y^{*}\right) \preccurlyeq^{U} F\left(x^{*}, y\right), \quad \forall x \in C, \quad y \in D .
$$


Definition 8 . Let $\left(X, \succcurlyeq^{X}\right),\left(Y, \succcurlyeq^{Y}\right)$ and $\left(U ; \succcurlyeq^{U}\right)$ be posets. Let $C$ and $D$ be nonempty subsets of $X$ and $Y$, respectively. Let $F: C \times D \rightarrow U$ be a mapping. A point $\left(x^{*}, y^{*}\right) \in C \times D$ is called an extended saddle point of the mapping $F$ if it satisfies

$$
F\left(x, y^{*}\right) \ngtr^{U} F\left(x^{*}, y^{*}\right) \ngtr^{U} F\left(x^{*}, y\right), \quad \forall x \in C, \quad y \in D .
$$

Definition 9. Let $\left(X, \succcurlyeq^{X}\right),\left(Y, \succcurlyeq^{Y}\right)$ and $\left(U ; \succcurlyeq^{U}\right)$ be posets. Let $C$ and $D$ be nonempty subsets of $X$ and $Y$, respectively. Let $F: C \times D \rightarrow U$ be a mapping. $F(x, y)$ is said to be

(1) order-nonnegative with respect to $x$, whenever for any $x_{2} \geqslant{ }^{X} x_{1}$ in $C, F\left(x_{1}, y\right) \nsucc^{U} F\left(x_{1}, t\right)$ implies $F\left(x_{2}, y\right) \ngtr^{U} F\left(x_{2}, t\right)$, for any $y, t \in D$;

(2) order-nonpositive with respect to $y$, whenever for any $y_{2} \geqslant^{Y} y_{1}$ in $C, F\left(x, y_{1}\right) k^{U} F\left(s, y_{1}\right)$ implies $F\left(x, y_{2}\right) k^{U} F\left(s, y_{2}\right)$, for any $x, s \in C$.

For a given mapping $F: C \times D \rightarrow U$, define $\gamma: C \rightarrow 2^{D}$ and $\zeta: D \rightarrow 2^{C}$ by

$$
\begin{aligned}
& \gamma(x) \\
& =\left\{t \in D: F(x, t) \ngtr^{U} F(x, y), \forall y \in D\right\}, \quad \text { for any } x \in C, \\
& \zeta(y) \\
& =\left\{s \in C: F(s, y) k^{U} F(x, y), \forall x \in C\right\}, \quad \text { for any } y \in D
\end{aligned}
$$

Theorem 10. Let $\left(X, \geqslant^{X}\right),\left(Y, \succcurlyeq^{Y}\right)$, and $\left(U ; \succcurlyeq^{U}\right)$ be posets. Let $C$ and $D$ be nonempty chain-complete subsets of $X$ and $Y$, respectively. Let $F: C \times D \rightarrow U$ be a mapping. If $F$ satisfies the following conditions,

(1) $F(x, y)$ is order-nonnegative with respect to $x \in C$;

(2) $F(x, y)$ is order-nonpositive with respect to $y \in D$;

(3) for every fixed $x \in C,\left(\gamma(x), \geqslant^{Y}\right)$ is a nonempty inductive subset of $D$ with finite number of maximal elements;

(4) for every fixed $y \in D,\left(\zeta(y), \geqslant^{X}\right)$ is a nonempty inductive subset of $C$ with finite number of maximal elements;

(5) there is an element $\left(x^{\prime}, y^{\prime}\right) \in C \times D$ with $x^{\prime} \preccurlyeq^{X} s$, for some $s \in \zeta\left(y^{\prime}\right)$ and $y^{\prime} \preccurlyeq^{Y}$ t and for some $t \in \gamma\left(x^{\prime}\right)$,

then $F$ has an extended addle point.

Proof. Define $T: C \times D \rightarrow 2^{C \times D} \backslash\{\varnothing\}$ by

$$
T(x, y)=\zeta(y) \times \gamma(x), \quad \forall(x, y) \in C \times D .
$$

From conditions (3) and (4) in this theorem, $T$ is well defined. Next we show that $T$ is order-increasing upward. To this end, we show that, for any $\left(x_{1}, y_{1}\right),\left(x_{2}, y_{2}\right) \in C \times$ $D,\left(x_{2}, y_{2}\right) \succcurlyeq^{X \times Y}\left(x_{1}, y_{1}\right)$ implies $T\left(x_{1}, y_{1}\right) \subseteq T\left(x_{2}, y_{2}\right)$. For any given $\left(s_{1}, t_{1}\right) \in T\left(x_{1}, y_{1}\right)$, we have $s_{1} \in \zeta\left(y_{1}\right)$ and $t_{1} \epsilon$ $\gamma\left(x_{1}\right)$; that is,

$$
\begin{aligned}
& F\left(s_{1}, y_{1}\right) \nless^{U} F\left(x, y_{1}\right), \quad \forall x \in C, \\
& F\left(x_{1}, t_{1}\right) \ngtr^{U} F\left(x_{1}, y\right), \quad \forall y \in D .
\end{aligned}
$$

Since $y_{2} \geqslant^{Y} y_{1}$, then, from condition (2) in this theorem that $F(x, y)$ is order-nonpositive with respect to $y \in D$. (14) implies $F\left(s_{1}, y_{2}\right) k^{U} F\left(x, y_{2}\right)$, for all $x \in C$. We obtain $s_{1} \in$ $\zeta\left(y_{2}\right)$. So

$$
\zeta\left(y_{1}\right) \subseteq \zeta\left(y_{2}\right)
$$

Similar to (16), for $x_{2} \geqslant^{X} x_{1}$, from condition (1) in this theorem, we can show that

$$
\gamma\left(x_{1}\right) \subseteq \gamma\left(x_{2}\right) .
$$

Combining (16) and (17), we get $\zeta\left(y_{1}\right) \times \gamma\left(x_{1}\right) \subseteq \zeta\left(y_{2}\right) \times \gamma\left(x_{2}\right)$. That is, $T\left(x_{1}, y_{1}\right) \subseteq T\left(x_{2}, y_{2}\right)$. It implies that $T$ is orderincreasing upward.

From the proof of Theorem 7 and conditions (3) and (4) in this theorem, we have that, for any $(x, y) \in C \times$ $D,\left(T(x, y), \geqslant^{C}\right)$ is an inductive poset with finite number of maximal elements. From condition (5) in this theorem, it is clearly seen that the element $\left(x^{\prime}, y^{\prime}\right) \in C \times D$ with $x^{\prime} \leqslant s$, for some $s \in \zeta\left(y^{\prime}\right)$ and $y^{\prime} \leqslant t$ and for some $t \in \gamma\left(x^{\prime}\right)$, satisfies $(s, t) \in \zeta\left(y^{\prime}\right) \times \gamma\left(x^{\prime}\right)=T\left(x^{\prime}, y^{\prime}\right)$ and $\left(x^{\prime}, y^{\prime}\right) \preccurlyeq^{X \times Y}(s, t)$. Hence the mapping $T$ satisfies all conditions in Theorem 2 with respect to condition $\left(\mathrm{A} 2^{\prime}\right)$. So $T$ has a fixed point; say $\left(x^{*}, y^{*}\right) \in C \times D$; that is, $\left(x^{*}, y^{*}\right) \in T\left(x^{*}, y^{*}\right)$. Then we have $x^{*} \in \zeta\left(y^{*}\right)$ and $y^{*} \in \gamma\left(x^{*}\right)$. So

$$
\begin{aligned}
& F\left(x^{*}, y^{*}\right) \star^{U} F\left(x, y^{*}\right), \quad \forall x \in C, \\
& F\left(x^{*}, y^{*}\right) \nsucc^{U} F\left(x^{*}, y\right), \quad \forall y \in D .
\end{aligned}
$$

That is

$$
F\left(x, y^{*}\right) x^{U} F\left(x^{*}, y^{*}\right) \chi^{U} F\left(x^{*}, y\right), \quad \forall x \in C, \quad y \in D .
$$

\section{Generalized and Extended Equilibrium Problems of Bifunctions on Posets}

Definition 11. Let $\left(X, \geqslant^{X}\right)$ and $\left(U ; \geqslant^{U}\right)$ be posets. Let $C$ be a nonempty subset of $X$. Let $F: C \times C \rightarrow U$ be a mapping. A point $x^{*} \in C$ is called a generalized equilibrium of the mapping $F$ if it satisfies

$$
F\left(x^{*}, x\right) \geqslant{ }^{U} F\left(x^{*}, x^{*}\right), \quad \forall x \in C .
$$

For a given mapping $F: C \times C \rightarrow U$, define $\Gamma: C \rightarrow 2^{C}$ by

$$
\Gamma(x)=\left\{t \in C: F(x, t)=\wedge_{y \in C} F(x, y)\right\}, \quad \text { for any } x \in C .
$$

We prove the following theorem for the existence of generalized equilibrium. The proof is similar to the proofs of Theorems 7 and 10. 
Theorem 12. Let $\left(X, \geqslant^{X}\right)$ and $\left(U ; \geqslant^{U}\right)$ be posets. Let $C$ be a nonempty chain-complete subset of $X$. Let F: $C \times C \rightarrow U$ be a mapping. If $F$ satisfies the following conditions,

(1) $F(x, y)$ is order-negative with respect to $x \in C$;

(2) for every fixed $x \in C,\left(\Gamma(x), \geqslant^{X}\right)$ is a nonempty inductive subset of $C$ with finite number of maximal elements;

(3) there is an element $x^{\prime} \in C$ with $x^{\prime} \preccurlyeq^{X} s$, for some $s \in$ $\Gamma\left(x^{\prime}\right)$,

then $F$ has a generalized equilibrium.

Proof. Taking the mapping $\Gamma$ defined by (20),

$$
\Gamma(x)=\left\{t \in C: F(x, t)=\wedge_{y \in C} F(x, y)\right\}, \quad \text { for any } x \in C .
$$

From condition (2) in this theorem, $\Gamma: C \rightarrow 2^{C} \backslash\{\varnothing\}$ is well defined.

Next we show that $\Gamma$ is order-increasing upward. To this end, we show that, for any $x_{1}, x_{2} \in C, x_{2} \geqslant{ }^{X} x_{1}$ implies $\Gamma\left(x_{1}\right) \subseteq$ $\Gamma\left(x_{2}\right)$. For any given $t_{1} \in \Gamma\left(x_{1}\right)$, we have

$$
F\left(x_{1}, t_{1}\right)=\wedge_{y \in D} F\left(x_{1}, y\right) .
$$

That is, $F\left(x_{1}, t_{1}\right) \preccurlyeq^{U} F\left(x_{1}, y\right)$, for all $y \in D$. Since $x_{2} \succcurlyeq^{X} x_{1}$, then, from condition (1) in this theorem that $F(x, y)$ is ordernegative with respect to $x \in C$, it implies $F\left(x_{2}, t_{1}\right) \preccurlyeq^{U} F\left(x_{2}, y\right)$, for all $y \in D$. That is, $F\left(x_{2}, t_{1}\right)=\wedge_{y \in D} F\left(x_{2}, y\right)$. We obtain $t_{1} \in \Gamma\left(x_{2}\right)$. Hence we have $\Gamma\left(x_{1}\right) \subseteq \Gamma\left(x_{2}\right)$. It implies that $\Gamma$ is order-increasing upward. From condition (3) in this theorem, it is clearly seen that the element $x^{\prime} \in C$ with $x^{\prime} \leqslant s$, for some $s \in \Gamma\left(x^{\prime}\right)$, satisfies $s \in \Gamma\left(x^{\prime}\right)$ and $x^{\prime} \preccurlyeq^{X}$ s. Hence the mapping $\Gamma$ from $C$ to $2^{C} \backslash\{\varnothing\}$ satisfies all conditions in Theorem 2 with respect to condition $\left(\mathrm{A} 2^{\prime}\right)$. So $\Gamma$ has a fixed point; say $x^{*} \in C$; that is, $x^{*} \in \Gamma\left(x^{*}\right)$. Then we have $F\left(x^{*}, x^{*}\right)=\wedge_{y \in C} F\left(x^{*}, y\right)$, which is equivalent to

$$
F\left(x^{*}, x\right) \geqslant F\left(x^{*}, x^{*}\right), \quad \forall x \in C .
$$

Definition 13. Let $\left(X, \geqslant^{X}\right)$ and $\left(U ; \geqslant^{U}\right)$ be posets. Let $C$ be a nonempty subset of $X$. Let $F: C \times C \rightarrow U$ be a mapping. A point $x^{*} \in C$ is called an extended equilibrium of the mapping $F$ if it satisfies

$$
F\left(x^{*}, x\right) k^{U} F\left(x^{*}, x^{*}\right), \quad \forall x \in C .
$$

It is clear to see that any generalized equilibrium of a mapping is an extended equilibrium of this mapping. For a given mapping $F: C \times C \rightarrow U$,define $\varphi$ : $C \rightarrow 2^{C}$ by

$$
\varphi(x)
$$$$
=\left\{t \in C: F(x, y) k^{U} F(x, t), \forall y \in C\right\}, \quad \text { for any } x \in C .
$$

Theorem 14. Let $\left(X, \succcurlyeq^{X}\right)$ and $\left(U ; \succcurlyeq^{U}\right)$ be posets. Let $C$ be a nonempty chain-complete subset of $X$. Let $F: C \times C \rightarrow U$ be a mapping. If $F$ satisfies the following conditions,

(1) $F(x, y)$ is order-nonnegative with respect to $x \in C$;

(2) for every fixed $x \in C,\left(\varphi(x), \geqslant^{X}\right)$ is a nonempty inductive subset of $C$ with finite number of maximal elements;

(3) there is an element $x^{\prime} \in C$ with $x^{\prime} \preccurlyeq^{X}$ s, for some $s \in$ $\varphi\left(x^{\prime}\right)$,

then $F$ has an extended equilibrium.

Proof. Taking the mapping $\varphi$ defined by (24),

$$
\begin{aligned}
& \varphi(x) \\
& \quad=\left\{t \in C: F(x, y) \star^{U} F(x, t), \forall y \in C\right\}, \quad \text { for any } x \in C .
\end{aligned}
$$

From condition (2) in this theorem, the mapping $\varphi: C \rightarrow$ $2^{C} \backslash\{\varnothing\}$ is well defined.

Next we show that $\varphi$ is order-increasing upward. To this end, we show that, for any $x_{1}, x_{2} \in C, x_{2} \geqslant{ }^{X} x_{1}$ implies $\varphi\left(x_{1}\right) \subseteq \varphi\left(x_{2}\right)$. For any given $t_{1} \in \varphi\left(x_{1}\right)$, we have $F\left(x_{1}, y\right) k^{U} F\left(x_{1}, t_{1}\right)$, for all $y \in C$. That is,

$$
F\left(x_{1}, t_{1}\right) \ngtr^{U} F\left(x_{1}, y\right), \quad \forall y \in C .
$$

Since $x_{2} \geqslant^{X} x_{1}$, then, from condition (1) in this theorem, $F(x, y)$ is order-nonnegative with respect to $x \in C$, and (26) implies $F\left(x_{2}, t_{1}\right) \ngtr^{U} F\left(x_{2}, y\right)$, for all $y \in C$. That is, $F\left(x_{2}, y\right) k^{U} F\left(x_{2}, t_{1}\right)$, for all $y \in C$. We obtain $t_{1} \in \varphi\left(x_{2}\right)$. So, we have $\varphi\left(x_{1}\right) \subseteq\left(x_{2}\right)$. Then the rest of the proof is similar to the proof of Theorem 12 .

\section{Generalized and Extended Ordered Variational Inequalities on Posets}

Let $\left(X, \geqslant^{X}\right)$ and $\left(U ; \succcurlyeq^{U}\right)$ be posets. We denote $M(X, U)$ for the collection of all mappings from $X$ to $U$.

Definition 15. Let $\left(X, \geqslant^{X}\right)$ and $\left(U ; \geqslant^{U}\right)$ be posets and $C$ a nonempty subset of $X$. Let $F: C \rightarrow M(X, U)$ be a mapping. A generalized ordered variational inequality problem associated with $C, F$, and $U$, denoted by $\operatorname{GOVI}(C, F, U)$, is to find a point $x^{*} \in C$, such that

$$
F\left(x^{*}\right)(x) \geqslant{ }^{U} F\left(x^{*}\right)\left(x^{*}\right), \quad \forall x \in C .
$$

Such a point $x^{*} \in C$ is called a generalized solution to the problem GOVI $(C, F, U)$.

Definition 16. Let $\left(X, \geqslant^{X}\right)$ and $\left(U ; \geqslant^{U}\right)$ be posets and $C$ a nonempty subset of $X$. Let $F: C \rightarrow M(X, U)$ be a mapping. $F$ is said to be order-negative on $C$, whenever, for any $x_{2} \geqslant x_{1}$ in $C, F\left(x_{1}\right)(y) \preccurlyeq^{U} F\left(x_{1}\right)(t)$ implies $F\left(x_{2}\right)(y) \preccurlyeq^{U} F\left(x_{2}\right)(t)$, for any $y, t \in X$. 
Theorem 17. Let $\left(X, \geqslant^{X}\right)$ and $\left(U ; \geqslant^{U}\right)$ be posets. Let $C$ be a nonempty chain-complete subset of $X$. Let $F: C \rightarrow M(X, U)$ be a mapping. If $F$ satisfies the following conditions,

(1) $F$ is order-negative on $C$;

(2) for every fixed $x \in C$, the poset $(\{t \in C: F(x)(t)=$ $\left.\left.\wedge_{y \in C} F(x)(y)\right\}, \geqslant^{X}\right)$ is a nonempty inductive subsetof $C$ with finite number of maximal elements;

(3) there is an element $x^{\prime} \in C$ with $x^{\prime} \preccurlyeq^{X} s$, for some $s \in C$ satisfying $F\left(x^{\prime}\right)(s)=\wedge_{y \in C} F\left(x^{\prime}\right)(y)$,

then the problem GOVI $(C, F, U)$ has a generalized solution.

Proof. Define a mapping $\Gamma$ by

$$
\Gamma(x)=\left\{t \in C: F(x)(t)=\wedge_{y \in C} F(x)(y)\right\}, \quad \text { for any } x \in C .
$$

From condition (2) in this theorem, $\Gamma: C \rightarrow 2^{C} \backslash\{\varnothing\}$ is well defined. Next we show that $\Gamma$ is order-increasing upward. To this end, we show that, for any $x_{1}, x_{2} \in C, x_{2} \geqslant{ }^{X} x_{1}$ implies $\Gamma\left(x_{1}\right) \subseteq \Gamma\left(x_{2}\right)$. For any given $t_{1} \in \Gamma\left(x_{1}\right)$, we have $F\left(x_{1}\right)\left(t_{1}\right)=$ $\wedge_{y \in C} F\left(x_{1}\right)(y)$. That is,

$$
F\left(x_{1}\right)\left(t_{1}\right) \preccurlyeq^{U} F\left(x_{1}\right)(y), \quad \forall y \in C .
$$

Since $x_{2} \geqslant^{X} x_{1}$, then, from condition (1) in this theorem that $F$ is order-negative on $C$, (29) implies $F\left(x_{2}\right)\left(t_{1}\right) \preccurlyeq{ }^{U} F\left(x_{2}\right)(y)$, for all $y \in C$. That is, $F\left(x_{2}\right)\left(t_{1}\right)=\wedge_{y \in C} F\left(x_{2}\right)(y)$. We obtain $t_{1} \in \Gamma\left(x_{2}\right)$. Hence we have $\Gamma\left(x_{1}\right) \subseteq \Gamma\left(x_{2}\right)$. It implies that $\Gamma$ is order-increasing upward. From condition (3) in this theorem, it is clearly seen that the element $x^{\prime} \in C$ with $x^{\prime} \leqslant s$, for some $s \in \Gamma\left(x^{\prime}\right)$, satisfies $s \in \Gamma\left(x^{\prime}\right)$ and $x^{\prime} \preccurlyeq^{X}$. Hence the mapping $\Gamma$ from $C$ to $2^{C} \backslash\{\varnothing\}$ satisfies all conditions in Theorem 2 with respect to condition $\left(\mathrm{A} 2^{\prime}\right)$. So $\Gamma$ has a fixed point; say $x^{*} \in C$; that is, $x^{*} \in \Gamma\left(x^{*}\right)$. We have $F\left(x^{*}\right)\left(x^{*}\right)=\wedge_{y \in C} F\left(x^{*}\right)(y)$. It is equivalent to

$$
F\left(x^{*}\right)(x) \geqslant{ }^{U} F\left(x^{*}\right)\left(x^{*}\right), \quad \forall x \in C .
$$

Definition 18. Let $\left(X, \geqslant^{X}\right)$ and $\left(U ; \succcurlyeq^{U}\right)$ be posets and $C$ a nonempty subset of $X$. Let $F: C \rightarrow M(X, U)$ be a mapping. An extended ordered variational inequality problem associated with $C, F$, and $U$, denoted by $(\operatorname{EOVI}(C, F, U))$, is to find a point $x^{*} \in C$, such that

$$
F\left(x^{*}\right)(x) k^{U} F\left(x^{*}\right)\left(x^{*}\right), \quad \forall x \in C . \quad(\operatorname{EOVI}(C, F, U))
$$

Such a point $x^{*} \in C$ is called an extended solution to the problem $(\operatorname{EOVI}(C, F, U))$.

Definition 19. Let $\left(X, \geqslant^{X}\right)$ and $\left(U ; \geqslant^{U}\right)$ be posets and $C$ a nonempty subset of $X$. Let $F: C \rightarrow M(X, U)$ be a mapping. $F$ is said to be order-nonnegative on $C$, whenever, for any $x_{2} \geqslant^{X} x_{1}$ in $C, F\left(x_{1}\right)(y) \nsucc^{U} F\left(x_{1}\right)(t)$ implies $F\left(x_{2}\right)(y) \ngtr^{U} F\left(x_{2}\right)(t)$, for any $y, t \in X$.
Theorem 20. Let $\left(X, \succcurlyeq^{X}\right)$ and $\left(U ; \succcurlyeq^{U}\right)$ be posets. Let $C$ be a nonempty chain-complete subset of $X$. Let $F: C \rightarrow M(X, U)$ be a mapping. If $F$ satisfies the following conditions,

(1) Fis order-nonnegative on $C$;

(2) for every fixed $x \in C,\left(\left\{t \in C: F(x, y) k^{U} F(x, t)\right.\right.$, for all $y \in C\}, \geqslant X)$, is a nonempty inductive subset of $C$ with finite number of maximal elements;

(3) there is an element $x^{\prime} \in C$ with $x^{\prime} \leqslant^{X} s$, for some $s \in C$ satisfying $F\left(x^{\prime}, y\right) k^{U} F\left(x^{\prime}, s\right)$, for all $y \in C$,

then the problem $(\operatorname{EOVI}(C, F, U))$ has an extended solution.

Proof. Define a mapping $\varphi$ by

$$
\begin{array}{r}
\varphi(x)=\left\{t \in C: F(x)(y) \aleph^{U} F(x)(t), \forall y \in C\right\}, \\
\text { for any } x \in C .
\end{array}
$$

From condition (2) in this theorem, $\varphi: C \rightarrow 2^{C} \backslash\{\varnothing\}$ is well defined. Next we show that $\varphi$ is order-increasing upward. To this end, we show that, for any $x_{1}, x_{2} \in C, x_{2} \geqslant{ }^{X} x_{1}$ implies $\varphi\left(x_{1}\right) \subseteq \varphi\left(x_{2}\right)$.For any given $t_{1} \in \varphi\left(x_{1}\right)$, we have $F\left(x_{1}\right)(y) k^{U} F\left(x_{1}\right)\left(t_{1}\right)$, for all $y \in C$. That is,

$$
F\left(x_{1}\right)\left(t_{1}\right) \ngtr^{U} F\left(x_{1}\right)(y), \quad \forall y \in C .
$$

Since $x_{2} \geqslant^{X} x_{1}$, then, from condition (1) in this theorem that $F$ is order-nonnegative on $C$, (32) implies $F\left(x_{2}\right)\left(t_{1}\right) \chi^{U} F\left(x_{2}\right)(y)$, for all $y \in C$. That is, $F\left(x_{2}\right)(y)$ $\aleph^{U} F\left(x_{2}\right)\left(t_{1}\right)$, for all $y \in C$. We obtain $t_{1} \in \varphi\left(x_{2}\right)$. Hence we obtain $\varphi\left(x_{1}\right) \subseteq \varphi\left(x_{2}\right)$. Then the rest of the proof is similar to the proof of Theorem 17 .

\section{Concluding Remarks}

In Sections 3 to 5 the existence of generalized and extended saddle points and equilibriums and the solvability of ordered variational inequalities are proved by applying fixed point Theorems 2 listed in Section 2 with respect to condition $\left(\mathrm{A} 2^{\prime}\right)$. As some special cases, the results in Sections 3 to 5 can be obtained by applying Theorem 2 with respect to condition $\left(\mathrm{A} 2^{\prime \prime}\right)$ or $\left(\mathrm{A} 2^{\prime \prime \prime}\right)$.

It is clearly seen that, from different fixed point theorems, one can obtain various results for solving some optimization problems. Note that all extensions of the Abian-Brown fixed point theorem listed in Section 2 are considered with chaincomplete posets. There are many examples in applications that the underlying spaces are not chain-complete. This aspect can be more precisely demonstrated by the following useful example.

For any positive integer $n$, let $\left(\mathbb{R}^{n} ; \geqslant^{n}\right)$ denote the $n$ dimensional poset where $\mathbb{R}^{n}$ is the $n$-dimensional Euclidean space equipped with the coordinate partial order $\geqslant^{n}$, which is defined as follows: for any $x=\left(x_{1}, x_{2}, \ldots, x_{n}\right), y=$ $\left(y_{1}, y_{2}, \ldots, y_{n}\right) \in \mathbb{R}^{n}, x \geqslant{ }^{n} y$ whenever $x_{j} \geq y_{j}$, for $j=$ $1,2, \ldots, n$. Then $\left(\mathbb{R}^{n} ; \geqslant^{n}\right)$ is not chain-complete, and it is conditionally chain-complete.

So if we are able to get some fixed point theorem on conditionally chain-complete posets, then we can study 
some optimization problems under more general underlying spaces: conditionally chain-complete posets, which include all problems studied in Sections 3 to 5 in this paper as special cases.

\section{Conflict of Interests}

The authors declare that there is no conflict of interests regarding the publication of this paper.

\section{Acknowledgments}

The authors greatly appreciate the anonymous referees' comments and suggestions, which improved the presentation of this paper.

\section{References}

[1] H. F. Bohnenblust and S. Karlin, "On a theorem of Ville," in Contributions to the Theory of Games, Annals of Mathematics Studies, no. 24, pp. 155-160, Princeton University Press, Princeton, NJ, USA, 1950.

[2] S. Carl and S. Heikkilä, Fixed Point Theory in Ordered Sets and Applications: From Differential and Integral Equations to Game Theory, Springer, New York, NY, USA, 2011.

[3] N.-J. Huang and Y.-P. Fang, "On vector variational inequalities in reflexive Banach spaces," Journal of Global Optimization, vol. 32, no. 4, pp. 495-505, 2005.

[4] I. V. Konnov and J. C. Yao, "On the generalized vector variational inequality problem," Journal of Mathematical Analysis and Applications, vol. 206, no. 1, pp. 42-58, 1997.

[5] J. von Neumann and O. Morgenstern, Theory of Games and Economic Behavior, Princeton University Press, Princeton, NJ, USA, 1944.

[6] C. J. Zhang, Set-Valued Analysis with Applications in Economics, Sciences Press, Beijing, China, 2004 (Chinese).

[7] F. Giannessi, "Theorems of alternative, quadratic programs and complementarity problems," in Variational Inequalities and Complementarity Problems, R. W. Cottle, F. Giannessi, and J. L. Lions, Eds., pp. 151-186, John Wiley \& Sons, Chichester, UK, 1980.

[8] J. Li, "Several extensions of the Abian-Brown fixed point theorem and their applications to extended and generalized Nash equilibria on chain-complete posets," Journal of Mathematical Analysis and Applications, vol. 409, no. 2, pp. 1084-1092, 2014.

[9] E. A. Ok, OrderTheory, forthcoming.

[10] L. Xie, J. Li, and W. Yang, "Order-clustered fixed point theorems on chain-complete preordered sets and their applications to extended and generalized Nash equilibria," Fixed Point Theory and Applications, vol. 2013, article 192, 2013.

[11] C. D. Aliprantis and O. Burkinshaw, Positive Operators, Springer, Dordrecht, The Netherlands, 2006.

[12] N. Dunford and J. T. Schwartz, Linear Operators-Part I, Wiley Classics Library, John Wiley \& Sons, New York, NY, USA, 1988.

[13] J. Li, "Applications of fixed point theory to generalized Nash equilibria of nonmonetized noncooperative games on Banach lattices," Nonlinear Analysis Forum, vol. 18, pp. 1-11, 2013. 


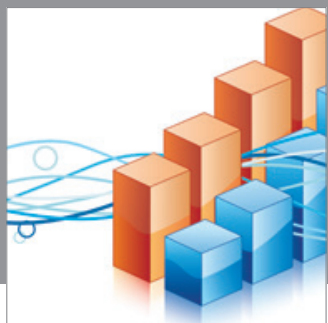

Advances in

Operations Research

mansans

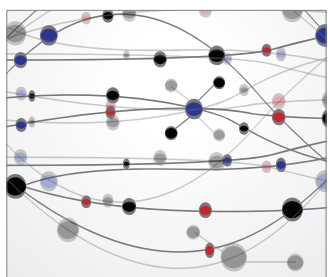

The Scientific World Journal
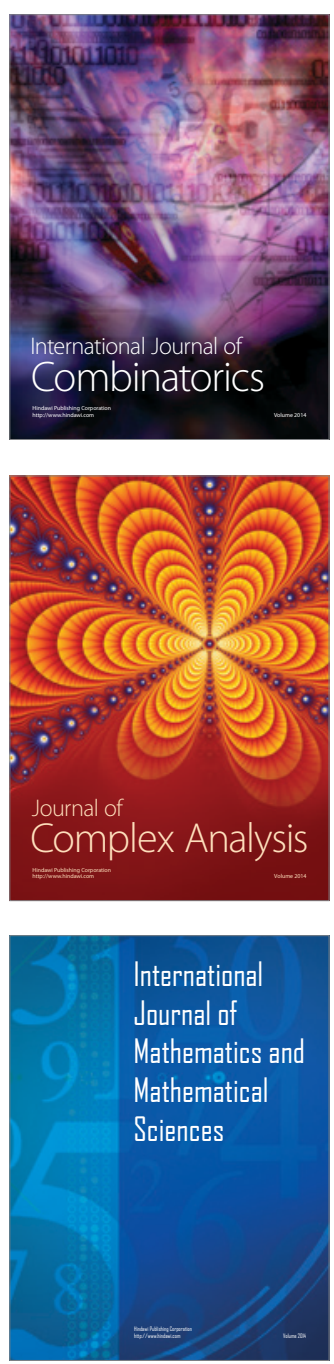
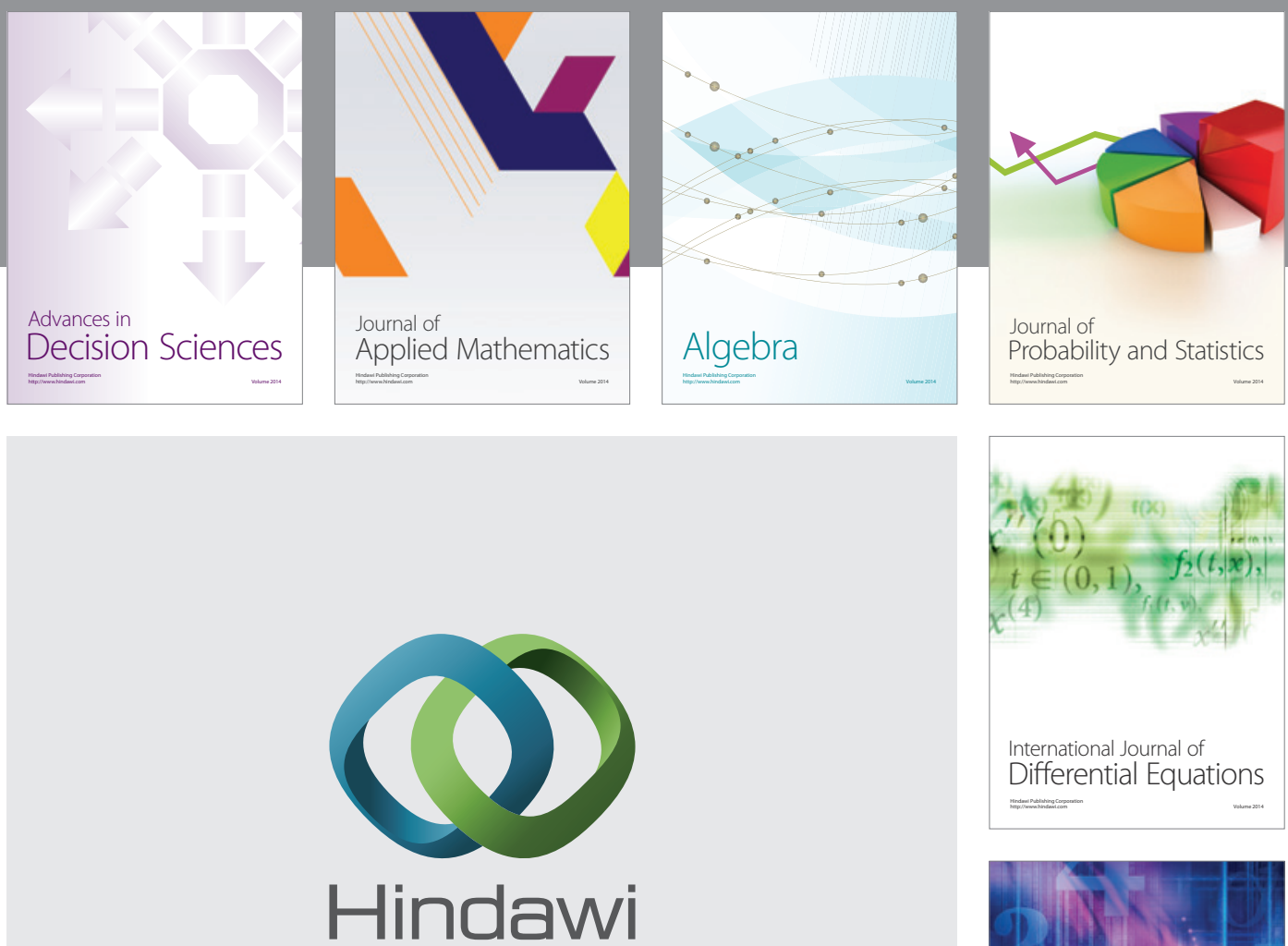

Submit your manuscripts at http://www.hindawi.com
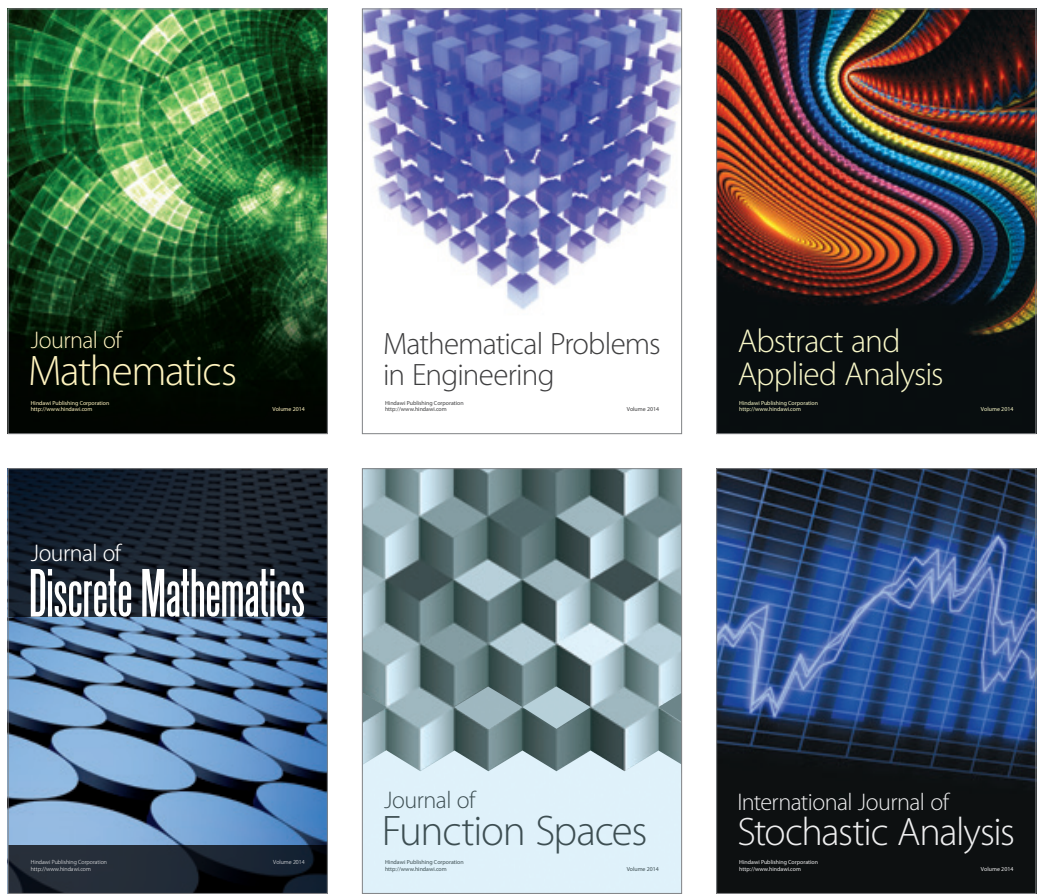

Journal of

Function Spaces

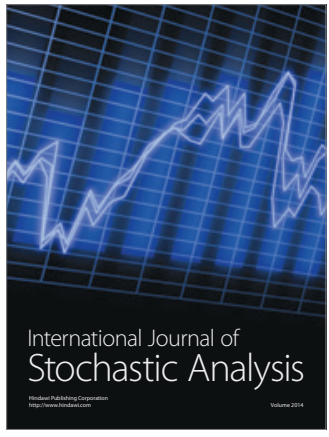

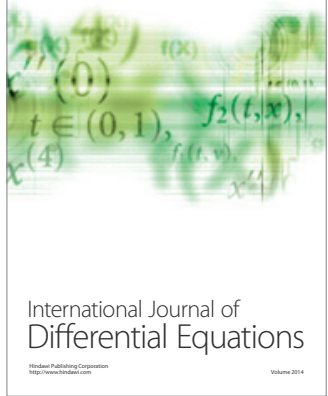
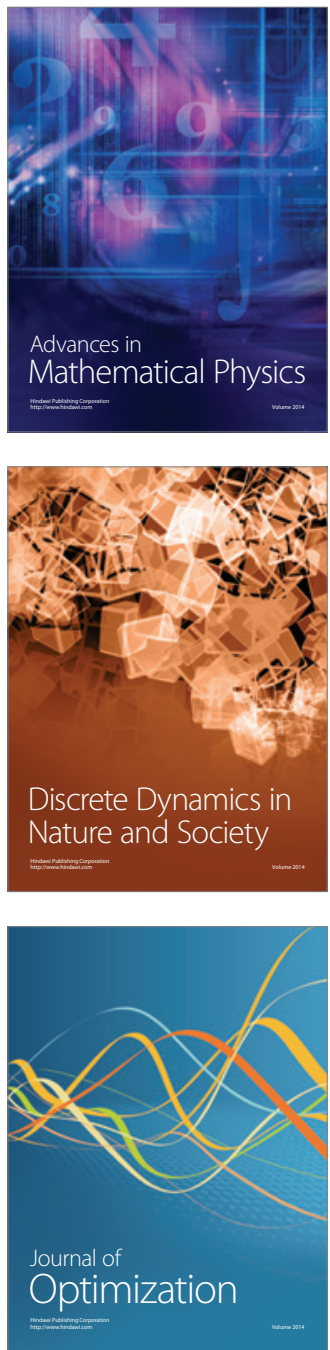